GLEMENTARY MIETHOD OF INVESTIGATING THE CENTROID, ETC.

\title{
Elementary Method of investigating the Centroid of a Uniform Circular Arc.
}

Let $A B$ and $B C$ be two circular arcs subtending angles $2 \alpha$ and $2 \beta$ at the common centre $O$. From symmetry the centroids $G_{1}, G_{2}$ and $G$ of $A B, B C$ and $A C$ lie on the bisectors $O P, O Q$ and

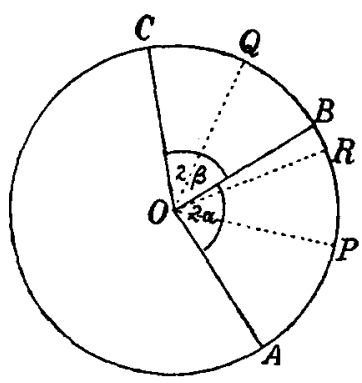

$O R$ of the angles which they subtend at the centre. Also, $G$ is the centroid of two particles placed at $G_{1}$ and $G_{2}$, and with masses proportional to the $\operatorname{arcs} A B$ and $B C$. Hence $G_{1}, G$, and $G_{2}$ are collinear, and

$$
\frac{G_{1} G}{G G_{2}}=\frac{\beta}{\alpha} .
$$

Now $\angle A O R=\angle R O C=\alpha+\beta$, therefore

$$
\text { and } \begin{aligned}
\angle P O R & =\angle A O R-\angle A O P=\beta, \\
\angle R O Q & =\angle R O C-\angle Q O C=\alpha .
\end{aligned}
$$

Hence $\quad \frac{G_{1} G}{G G_{2}}=\frac{O G_{1}}{O G_{2}} \cdot \frac{\sin G_{1} O G}{\sin G O G_{2}}=\frac{O G_{1} \sin \beta}{O G_{2} \sin \alpha}$.

Equating (1) and (2) we have

$$
O G_{1}: O G_{2}=\frac{\sin \alpha}{\alpha}: \frac{\sin \beta}{\beta} .
$$

Hence the ratio $O G_{1}: \frac{\sin \alpha}{\alpha}$ is independent of $\alpha$, and therefore

$$
O G_{1}=k \frac{\sin \alpha}{\alpha}=k \frac{\text { chord }}{\operatorname{arc}},
$$

the angle $\alpha$ being in circular measure. 
To find $k$, let the are diminish and tend to zero, then the ratio chord $:$ arc $\rightarrow 1$, and $O G_{1} \rightarrow$ the radius, hence finally

$$
O G=\operatorname{radius} \times \frac{\text { chord }}{\text { arc }} \text {. }
$$

D. M. Y. SOMMERVILLE.

\section{* Nomogram for the Solution of the Equation

$$
\boldsymbol{z}^{3}+\boldsymbol{p} \boldsymbol{z}+\boldsymbol{q}=0 .
$$

The curve $y=x^{3}$ is constructed with a scale of $l^{\prime \prime}$ horizontally and $\frac{1}{10}$ " vertically. It is graduated with the values of $x$. Then draw the two lines $x= \pm 1$ and graduate them on the scale of the $y$-axis, $x=+1$ positive downwards, $x=-1$ positive upwards.

To solve the equation $z^{3}+p z+q=0$, set $p+q$ on the line $x=+1$ and $p-q$ on the line $x=-1$. Join the two points and get the intersections with the curve. The accompanying diagram has only a range between \pm 3.5 , but it is easy to divide the roots by 2,3 , or 10 . If accurately drawn, it should give two figures of the root with ease, as a rule, and may give three. Sometimes, of course, it may be difficult to separate the roots, e.g. in the equation $z^{3}-13 z+18=0$. The line seems to touch about $+2 \cdot 2$, and the 3rd root is off the paper. Dividing the roots by 2, we get $z^{3}-3 \cdot 25 z+2 \cdot 25=0$. We see that 1 is a root, another root is $-2 \cdot 08$, and therefore the third root is $+1 \cdot 08$. Hence the roots of the given equation are $2,2 \cdot 16,-4 \cdot 16$.

I find it convenient to use, instead of a ruler, which hides part of the diagram, a strip of celluloid with a fine line ruled on the lower surface.

D. M. Y. Sommerville. 